\title{
BORRELIA BURGDORFERI SENSU LATO, THE AGENT OF LYME BORRELIOSIS: LIFE IN THE WILDS
}

GERN L.*

\section{Summary :}

In Europe, Borrelia burgdorferi sensu lato (sl) the agent of Lyme borreliosis circulates in endemic areas between Ixodes ricinus ticks and a large number of vertebrate hosts upon which ticks feed. Currently, at least 12 different Borrelia species belonging to the complex B. burgdorferi sl have been identified among which seven have been detected in I. ricinus: B. burgdorferi sensu stricto (ss), B. garinii, B. afzelii, B. valaisiana, B. spielmanii and B. bissettii. A few dozens of vertebrate hosts have been identified as reservoirs for these Borrelia species. Specific associations were rather early observed between hosts, ticks and borrelia species, like for example between rodents and B. afzelii and

B. burgdorferi ss, and between birds and B. garinii and

$B$. valaisiana. The complement present in the blood of the hosts is the active component in the Borrelia host specificity. Recent studies confirmed trends toward specific association between Borrelia species and particular host, but also suggested that loose associations may be more frequent in transmission cycles in nature than previously thought.

KEY WORDS : Ixodes ricinus, host DNA, Borrelia, reservoir hosts.

工 $\mathrm{n}$ n Europe, Lyme borreliosis endemic areas are maintained through complex interactions among different tick species, a variety of Borrelia strains belonging to the complex B. burgdorferi sensu lato (sl) and a large number of vertebrate hosts upon which ticks feed.

The tick I. ricinus is the classical tick vector in the European distribution of Lyme borreliosis. I. ricinus is very generalist feeding on numerous mammalian, avian and reptilian species in the European woodlands. This tick species is a three-host tick that goes through three developmental stages: larva, nymph, and male and female adults. To encounter its hosts, I. ricinus quests on the tip of low vegetation. During questing, ticks very often have to face desiccating conditions and have to quit their questing place to move to the litter zone where they regain lost body water (Randolph \& Storey, 1999). Interruption of questing occurs mostly during darkness (Perret et al., 2003). Interestingly, I. ricinus moves preferentially when desiccation risk is the lowest in nature,

\footnotetext{
* Institute of Biology, University of Neuchâtel, Switzerland, Emile Argand 11, Case postale 158, 2009 Neuchâtel, Switzerland.

Tel.: +41327183000 - Fax: +41327183001.

E-mail: lise.gern@unine.ch
}

that is to say at sundown. The change in light intensity that triggers tick movements is perceived by I. ricinus thanks to photosensitive cells (Perret et al., 2003). Under moisture stress, ticks will quest for shorter periods and this will decrease the host-finding probability and thus influences tick population dynamic (Perret et al., 2003). If high desiccating conditions are lasting too long, tick mortality is increased resulting in questing tick population decrease (Perret et al., 2000, 2004).

Besides I. ricinus, other tick species have been found infected by spirochetes but only $I$. hexagonus, that feeds mainly on carnivores, and I. uriae, that is associated with seabirds, are able to support natural transmission of Borreliae and to significantly contribute to the persistence of spirochetes within endemic areas (Gern et al., 1997; Olsen et al., 1993). Spirochetes have also been detected or isolated from other tick species and also from insects, especially from mosquitoes and fleas but their role in the maintenance of a focus remains unknown (Gern \& Humair, 2002).

At least 237 animal species are implicated, as hosts for ticks, in the maintenance of I. ricinus tick populations and, for this reason, are susceptible to serve as reservoir hosts for Borrelia. Nevertheless, compared to the large number of tick hosts, little information is available on the real significance of most animal hosts as sources for infecting $I$. ricinus with B. burgdorferi sl. Tick xenodiagnosis is the only way to really demonstrate the infectivity of a vertebrate species. Xenodiagnosis consists in allowing naive ticks derived from a laboratory colony free of infection to engorge on tested hosts and to analyze the ticks after the meal or after the moult to detect the pathogen. This technique also allows identify the Borrelia species transmitted from the vertebrate to the ticks. However, xenodiagnosis, without counting that a laboratory colony of ticks has to be available, is a very fastidious method because hosts have to be captured and maintained in captivity. Hence, it is easy to understand that small mammals are clearly the vertebrate group the most extensively studied. Currently, the infectivity of Apodemus mice and Clethrionomys voles for $I$. ricinus ticks is unanimously recognized (Gern \& Humair, 2002). If the role of small 
rodents in the circulation of Borrelia in nature was rather easily elucidated, controversy long surrounded the role of birds as reservoirs. Today, the reservoir role of various bird species is unanimously admitted, especially concerning ground-nesting and ground-foraging birds - like thrushes, blackbirds, robins, wrens, nightingales, blue throats and pheasants. Migratory birds have also been shown through their infectivity for I. ricinus to participate in the enzootic maintenance of $B$. burgdorferi sl, and their role in the spread of infected ticks is now also well documented (Comstedt et al., 2006; Olsen et al., 1995; Poupon et al., 2006). Studies on hares, squirrels and hedgehogs revealed that they contribute to Borrelia infection in ticks (Gern \& Humair, 2002). Among large size mammals, red foxes are also able to infect ticks with Borrelia but the infectivity of red foxes appears as low. While the reservoir role of various tick hosts species was being identified, the role of largesized mammals like ungulates in the contribution of the infection of ticks was denied although their role as hosts for ticks, particularly adult ticks, is considerable. In recent years, the taxonomy of $B$. burgdorferi sl spirochetes has become complicated and at least 12 different Borrelia species have been identified among which seven are circulating in endemic areas in Europe and have been detected in I. ricinus: B. burgdorferi sensu stricto (ss), B. garinii, B. afzelii, B. valaisiana, B. spielmanii and B. bissettii (Rauter \& Hartung, 2005; Richter et al., 2006). Only one study reported $B$. bissettii in $I$. ricinus (Hanincova et al., 2003) and therefore its presence in this tick vector remains to be confirmed.

The description of the various Borrelia species in ticks has opened up an entire new field of research in the ecology of Lyme borreliosis. A number of key issues arose from this situation and the relation existing between the various Borrelia species and the hosts had to be examined in more details. Specific associations were rather early observed between hosts, ticks and borreliae species that help us today to better understand the ecology of B. burgdorferi. First studies strongly suggested that a specific association existed between small mammals and B. afzelii. Then B. burgdorferi ss and a subtype of $B$. garinii were also associated with rodents. In addition, the frequent observations of $B$. burgdorferi ss and $B$. afzelii in the skin of grey and red squirrels and the high prevalence of these two Borrelia species in ticks collected from the squirrels suggested that B. burgdorferi ss and B. afzelii were transmitted from squirrels to feeding ticks. The first report of $B$. garinii in bird feeding ticks implied that B. garinii might be associated with birds. This was confirmed later for pheasants and blackbirds, and B. valaisiana was also implicated in this association with birds. The situation with $B$. lusitaniae is especially interesting. In fact, quite recently, very different vertebrate groups, such as lizards, birds and hedgehogs, were identified as reservoirs for B. lusitaniae (Amore et al., 2007; Dsouli et al., 2006; Poupon et al., 2006; Richter \& Matuschka, 2006; Skuballa et al., 2007). Eventually, concerning the more recent identified Borrelia species, B. spielmanii, little is known on the reservoir hosts. Only one study reported two species of dormouse as reservoirs for this species (Richter et al., 2004). In other words, various studies in endemic areas throughout Europe reported associations between some Borrelia species and particular groups of vertebrate hosts. The explanation to these associations was given by researches summarized in Kurtenbach et al. (2002). The complement present in the blood of the hosts was shown to be the active component in the Borrelia host specificity. This information provided new insights into the dynamics of the circulation of the various Borrelia species among tick host populations. In vitro tests demonstrated that the pattern of serum sensitivity of different Borrelia species matched the known reservoir status of many vertebrate species for $B$. burgdorferi. For example, B. afzelii was resistant to rodent serum whereas $B$. garinii was destroyed by rodent serum (Kurtenbach et al., 2002). The absence of generalized infection in deer correlated with the indiscriminatory borreliacidal activity of deer sera against all the Borrelia species tested and explained the fact that this host does not play a role in the maintenance of Borrelia in endemic areas as already mentioned. However, it seems that the situation is not clear-cut and that some exceptions exist, and that looser associations are present in nature. In fact, results from other studies conflicted with this situation, for example not only B. afzelii or $B$. burgdorferi ss but also additional Borrelia species and subtypes were isolated or PCR-detected in small mammals or in xenodiagnostic ticks fed on small rodents (Korenberg et al., 2002). Similarly, B. afzelii DNA was detected in xenodiagnostic ticks fed on blackbirds (Humair et al., 1999). Differences in biological and ecological factors such as different Borrelia subtypes, different tick vector species and different veretebrate biocenosis present in different areas could explain the discrepancy observed. The situation with $B$. lusitaniae is especially interesting. As reported by Kurtenbach et al. (2002), exposure of B. lusitaniae to lizard and bird serum resulted in killing of spirochetes. Curiously, as mentioned before, lizards and birds have been identified as reservoir hosts for $B$. lusitaniae in nature. All this strongly suggests that still much more efforts have to be put on the investigation of the role of vertebrates in the infection of ticks in local areas, keeping in mind the existence of several species and subtypes of B. burgdorferi sl. with apparently different host specificities. This is supported by results obtained by more sophisticated methods recently developed to assess the reservoir role of the various tick hosts. In fact, thanks to implementation of more performing molecular analysis techniques, it is currently possible to identify the hosts 
that have fed the field-collected ticks in their previous developmental stages and to simultaneously detect tickborne pathogens in the same ticks. Kirstein \& Gray (1996) were the first to demonstrate the feasibility to identify host DNA in ticks by using a PCR targeting part of the cytochrome B gene. Later, studies targeting the nuclear 18S rRNA gene (Pichon et al., 2003) and the $12 \mathrm{~S}$ rDNA mitochondrial gene (Humair et al., 2007) were developed. The method based on nuclear $18 \mathrm{~S}$ rRNA gene appears as less sensitive in the sense that this method allows discrimination only among major groups of vertebrate hosts whereas the other method based on the 12S rDNA mitochondrial gene has the advantage to allow host DNA identification at the species level (Humair et al., 2007). The $12 \mathrm{~S}$ rDNA mitochondrial gene based method permits a large panel of host identification since approximately 30 different vertebrate species DNA can be detected. However, one should stress here that these methods are limited by their sensitivity. None of them globally allowed higher identification success than $50 \%$. Of course, the main drawbacks to these approaches are the small quantities of blood meal remnants still present in questing ticks and the time passed since the last blood meal. In fact one should keep in mind that ticks can quest for months on vegetation and hence, analysed host DNA in tick midgut is most of the time several months old. Both methods have been used to identify hosts for ticks in various areas in Europe and also to identify reservoir hosts for Borrelia and other tick-borne pathogens (Pichon et al., 2005; 2006, Estrada-Pena et al., 2005; Moràn Cadenas et al., 2007). All these studies confirmed trends toward specific association between Borrelia species and particular host but also suggested that loose associations may be more frequent in transmission cycles in nature than previously thought.

In conclusion, it seems prudent to review the importance of the different type of associations between Borrelia species in various geographic areas. Clearly, additional field studies are expected in the subject of Borrelia host specificities, keeping in mind, particularly the rare Borrelia species and the huge subtype diversity hidden behind most Borrelia species, as well as the geographic diversity of local ecosystems. Therefore, future studies in Lyme borreliosis ecology seeking to identify the Borrelia species found in both ticks and reservoir hosts in various endemic areas are strongly encouraged.

\section{REFERENCES}

Amore G., Tomassone L., Grego E., Ragagli C., Bertolotti L., Nebbia P., Rosati S. \& Mannelli A. Borrelia lusitaniae in immature Ixodes ricinus (Acari: Ixodidae) feeding on common wall lizards in Tuscany, central Italy. Journal of Medical Entomology, 2007, 44, 303-307.
Comstedt P., Bergström S., Olsen B., Garpmo U., Marjavaara L., Mejlon H., Barbour A.G. \& Bunikis J. Migratory passerine birds as reservoirs of Lyme borreliosis in Europe. Emerging Infectious Diseases, 2006, 12, 1087-1095.

Dsouli N., Younsi-Kabachit H., Postic D., Nouira S., Gern L. \& Bouattour A. Reservoir role of the lizard, Psammodromus algirus, in the transmission cycle of Borrelia burgdorferi sensu lato (Spirochaetacea) in Tunisia. Journal of Medical Entomology, 2006, 43, 737-742.

estrada-Pena A., Osacar J.J., Pichon B. \& Gray J.S. Hosts and pathogen detection for immature stages of Ixodes ricinus (Acari: Ixodidae) in North-Central Spain. Experimental and Applied Acarology, 2005, 37, 257-268.

GERN L. \& HuMAIR P.F. Ecology of Borrelia burgdorferi sensu lato in Europe, in: Lyme borreliosis: biology, epidemiology and control. Gray J.S., Kahl O., Lane R.S., Stanek G. (eds.), CAB International, Wallingford, Oxon, United Kingdom, 2002, 149-174.

Gern L., Rouvinez E., Toutoungi, L.N. \& Godfroid E. Transmission cycles of Borrelia burgdorferi sensu lato involving Ixodes ricinus and/or I. hexagonus ticks and the European hedgehog, Erinaceus europaeus, in suburban and urban areas in Switzerland. Folia Parasitologica, 1997, 44, 309-314.

Hanincová K., Taragelová V., Koci J., Schäfer S.M., Hails R., Ullmann A.J., Piesman J., Labuda M. \& Kurtenbach K. Association of Borrelia garinii and B. valaisiana with songbirds in Slovakia. Applied Environmental Microbiology, 2003, 69, 2825-2830.

Humair P.F., Postic D., Wallich R. \& Gern L. An avian reservoir (Turdus merula) of the Lyme borreliosis spirochetes. Zentralblatt für Bakteriologie, 1999, 287, 521-538.

Humair P.F., Douet V., Morán Cadenas F., Schouls L., VAN DE POL I. \& GERN L. Molecular identification of blood meal source in Ixodes ricinus ticks using $12 \mathrm{~S}$ rDNA as a genetic marker. Journal of Medical Entomology, 2007, 44, 869-880.

Kirstein F. \& Gray J.S. A molecular marker for the identification of the zoonotic reservoirs of Lyme borreliosis by analysis of the blood meal in its European vector Ixodes ricinus. Applied Environmental Microbiology, 1996, 62, 4060-4065.

Korenberg E.E., Gorelova N.B. \& Kovalevskit Y.V. Ecology of Borrelia burgdorferi sensu lato in Russia, in: Lyme borreliosis: biology, epidemiology and control. Gray J.S., Kahl O., Lane R.S., Stanek G. (eds), CAB International, Wallingford, Oxon, United Kingdom, 2002, 175-200.

Kurtenbach K., Schäfer S.M., de Michelis S., EtTi S. \& Sewell H.S. Borrelia burgdorferi sensu lato in the vertebrate host, in: Lyme borreliosis: biology, epidemiology and control. Gray J.S., Kahl O., Lane R.S., Stanek G. (eds.), CAB International, Wallingford, Oxon, United Kingdom, 2002, 117-150.

Morán Cadenas F., Rais O., Jouda F., Humair P.F., Douet V., MORET J. \& GERN L. Identification of host bloodmeal source and Borrelia burgdorferi sensu lato in field-collected Ixodes ricinus ticks in Chaumont (Switzerland). Journal of Medical Entomology, 2007, in press.

Olsen B., Jaenson T.G.T., Noppa L., Bunikis J. \& Bergström S. A Lyme borreliosis cycle in seabirds and Ixodes uriae ticks. Nature, 1993, 362, 340-342. 
Olsen, B., Jaenson T.G.T. \& Bergström S. Prevalence of Borrelia burgdorferi sensu lato-infected ticks on migrating birds. Applied and Environmental Microbiology, 1995, 61, 3082-3087.

Perret J.L., Guerin P.M., Diehl P.A., Vlimant M. \& Gern L. Darkness induces mobility, and saturation deficit limits questing duration, in the tick Ixodes ricinus. Journal of Experimental Biology, 2003, 206, 1809-1815.

Perret J.L., Guigoz E., Rais O. \& Gern L. Influence of saturation deficit and temperature on Ixodes ricinus tick questing activity in a Lyme borreliosis endemic area (Switzerland). Parasitology Research, 2000, 86, 554-557.

Perret J.L., Rais O. \& Gern L. Influence of climate on the proportion of Ixodes ricinus nymphs and adults questing in a tick population. Journal of Medical Entomology, 2004, 41, 361-365.

Pichon B., Egan D., Rogers M. \& Gray J.S. Detection and identification of pathogens and host DNA in unfed hostseeking Ixodes ricinus L. (Acari: Ixodidae). Journal of Medical Entomology, 2003, 40, 723-731.

Pichon B., Rogers M., Egan D. \& Gray J. Blood-meal analysis for the identification of reservoir hosts of tick-borne pathogens in Ireland. Vector Borne Zoonotic Diseases, 2005, 5, 172-180.

Pichon B., Kahl O., Hammer B. \& Gray J.S. Pathogens and host DNA in Ixodes ricinus nymphal ticks from a German forest. Vector Borne Zoonotic Diseases, 2006, 6, 382-387.

Poupon M.A., Lommano E., Humair P.F., Douet V., Rais O., SchaAd M., Jenni L. \& Gern L. Prevalence of Borrelia burgdorferi sensu lato in ticks collected from migratory birds in Switzerland. Applied Environmental Microbiology, 2006, 72, 976-979.

RANDOLPH S.E. \& STOREY K. Impact of microclimate on immature tick-rodent host interactions (Acari: Ixodidae): implications for parasite transmission. Journal of Medical Entomology, 1999, 36, 741-748.

RAUTER C. \& HaRtung T. Prevalence of Borrelia burgdorferi sensu lato species in Ixodes ricinus ticks in Europe: a metaanalysis. Applied Environmental Microbiology, 2005, 71, 7203-7216.

Richter D. \& Matuschka F.R. Perpetuation of the Lyme disease spirochete Borrelia lusitaniae by lizards. Applied Environmental Microbiology, 2006, 72, 4627-4632.

Richter D., Postic D., Sertour N., Livey I., Matuschka F.R. \& BARANTON G. Delineation of Borrelia burgdorferi sensu lato species by multilocus sequence analysis and confirmation of the delineation of Borrelia spielmanii sp. nov. International Journal of Systematic Evolution and Microbiology, 2006, 56, 873-881.

Richter D., Schlee D.B., Allgöver R. \& MatuschKa F.R. Relationships of a novel Lyme disease spirochete, Borrelia spielmani sp. nov., with its hosts in Central Europe. Applied Environmental Microbiology, 2004, 70, 6414-6419.

Skuballa J., Oehme R., Hartelt K., Petney T., Bücher T., Kimmig P. \& TARASChewski H. European hedgehogs as hosts for Borrelia spp., Germany. Journal of Emerging Diseases, 2007, 13, 952-953. 